\title{
Deshidratación Osmótica y Secado por Aire Caliente en Mango, Guayaba y Limón para la Obtención de Ingredientes Funcionales
}

Hilda H. Estrada ${ }^{(1)^{\star}}$, Claudia E. Restrepo ${ }^{(2)}$, Hernán G. Saumett ${ }^{(1)}$ y Liliana Pérez ${ }^{(3)}$

(1) Universidad Simón Bolívar de Barranquilla, Facultad de Administración y Negocios, Carrera 65 No. 64-17

Barranquilla- Colombia (e-mail: hileslo@hotmail.com)

(2) Instituto de Ciencia y Tecnología Alimentaria - INTAL, Carrera 50G No 12 sur-91 Itagüí - Colombia.

(e-mail: crestrepo@intal.org)

(3) Universidad Simón Bolívar de Barranquilla, Facultad de Ciencias Básicas y Biomédicas, Barranquilla-

Colombia. (e-mail: Iperez70@unisimonbolivar.edu.co)

${ }^{*}$ Autor a quien debe ser enviada la correspondencia

Recibido Nov. 9, 2017; Aceptado Ene. 16, 2017; Versión final Feb. 19, 2018, Publicado Jun. 2018

\section{Resumen}

Se presenta y se discute los resultados de la aplicación de técnicas de deshidratación osmótica y secado por aire caliente en mango, guayaba y limón procedentes de pequeños productores agropecuarios del Departamento de Atlántico-Colombia como estrategia para dar valor agregado y aprovechamiento a sus cosechas a través de la obtención de ingredientes funcionales. Se determinaron compuestos de interés nutraceútico: fibra, calcio y vitamina $C$ antes y después de los procesos de deshidratación. Se obtuvieron productos de humedad baja comprendidos por ralladura de limón deshidratada, lámina de mango-limón, mango en polvo, guayaba en polvo y de humedad intermedia comprendido por mango y guayaba osmodeshidratada. La técnica de secado por aire caliente permitió obtener ralladura de cascara de limón, mango y guayaba en polvo, con contenidos importantes de fibra dietaria y los dos últimos también de vitamina $\mathrm{C}$, en contraste con una perdida drástica de vitamina $\mathrm{C}$ en los productos osmo-deshidratados.

Palabras Clave: deshidratación aire caliente; osmodeshidratación; mango; guayaba; limón; vitamina C.

\section{Osmotic Dehydration and Hot Air Drying on Mango, Guava and Lemon to Obtain Functional Ingredients}

\begin{abstract}
The main objective of this paper is to present and discuss the results of the application of the techniques of osmotic dehydration and hot air drying on mango, guava and lemon so as to give them an added value and to make the most out of these crops to obtain functional ingredients. These fruits came from small agricultural producers in the Department of Altantico, Colombia. The compounds of nutraceutical interest were determined: fiber, calcium and vitamin C. All of them were studied before and after the dehydration process. These two different products were obtained: of low humidity, such as lemon dehydrated peel zest, mango-lemon sheet, mango powder, and guava powder; and of intermediate humidity, dehydrated mango and guava. The hot airdrying process allowed to obtain a lemon dehydrated peel zest, mango powder, and guava powder. These last two products contained an important amount of dietary fiber and vitamin C. By contrast, there was a significant loss of vitamin $\mathrm{C}$ in the osmodehydrated products.
\end{abstract}

Keywords: hot air drying; osmotic dehydration; mango; guava; lemon; vitamin C 


\section{INTRODUCCIÓN}

Los alimentos funcionales y los suplementos representan una oportunidad para el diseño y formulación de alimentos orientado a productos que contribuyan a conservar la salud y a prevenir enfermedades; mediante la incorporación de materias primas o ingredientes con actividades funcionales (Pérez, 2006). La tendencia mundial se orienta hacia la incorporación de productos nutracéuticos, los cuales son sustancias químicas o biológicas que pueden encontrarse como componentes naturales de los alimentos o adicionarse a los mismos y que resultan especialmente beneficiosas (Diaz et al., 2017; Pérez, 2006). Por lo anterior, la búsqueda de alimentos funcionales y nutracéuticos son un reto para la ciencia y tecnología de los alimentos. Entre los productos e ingredientes potencialmente aprovechables, se encuentran las frutas y hortalizas, que constituyen una fuente potencial de antioxidantes, micronutrientes, vitaminas, carbohidratos, minerales, bioactivos, entre otros (Anany, 2015; Contreras-Calderón et al., 2011; Vergara-Valencia et al., 2007; Zapata et al., 2013). Las frutas además contienen una alta proporción de agua y la distribución de los compuestos bioactivos y su estabilidad se ve fuertemente afectada por los procesos de almacenamiento; por lo cual, para su conservación se hace necesario utilizar técnicas de deshidratación que permitan concentrar los compuestos de interés y conservar algunas de sus características sensoriales, críticas para la comercialización (Erenturk et al., 2005; Fernández et al., 2015; Pereira et al., 2013). La vitamina C suele seleccionarse como un índice de la calidad de los nutrientes debido a su naturaleza lábil en comparación con los otros nutrientes en los alimentos. La degradación del ácido ascórbico depende de varios factores, que incluyen oxígeno, catálisis de iones metálicos, luz, temperatura y contenido de humedad, por lo que la vitamina $\mathrm{C}$ se considera como un indicador de la calidad en la elaboración de alimentos debido a su baja estabilidad durante los procesos térmicos (Erenturk et al., 2005; Mendoza et al., 2015).

Entre las técnicas de deshidratación de frutas con mayor proyección, se encuentra la deshidratación osmótica, la cual consiste en sumergir trozos en una solución hipertónica (solución osmótica) compuesta por solutos capaces de generar una presión osmótica alta, con una doble transferencia de masa (agua de la fruta a la solución y solutos de la solución a la fruta) (Ahmed et al., 2016). Este proceso depende de la geometría del producto y de las propiedades de la solución osmótica, con temperaturas entre $40-80^{\circ} \mathrm{C}$ (Chandra y Kumari, 2015; Giraldo-Bedoya et al., 2004; Pereira et al., 2013; Alvis et al., 2016) ; pudiendo obtener productos con propiedades de calidad adecuada (Fernández et al., 2015; Molina y Orjuela, 2013; Tortoe, 2010). Otra alternativa es la deshidratación por flujo de aire caliente, en la cual, a partir de la evaporación se elimina el contenido de agua y se impide el crecimiento bacteriano (Serpa-Guerra et al., 2015; Vega-Gálvez et al., 2009). En el secado de frutas mediante este proceso se pueden afectar las propiedades sensoriales y el valor nutricional si se almacena a temperaturas muy altas. La temperatura de secado es una variable fundamental en los estudios cinéticos de cualquiera de los procesos (Pereira et al., 2013).

El limón, el mango y la guayaba son las frutas más producidas en la región del sur del Atlántico en Colombia, estas frutas presentan propiedades nutracéuticas de interés: el limón contiene principalmente vitamina $\mathrm{C}$ y compuestos fenólicos (flavonoides y ácidos hidroxicinámicos), que proporcionan funciones antivirales, antialérgicas, antiinflamatorias y anticancerígenas; el mango por su parte, es una excelente fuente de vitaminas como el ácido ascórbico, tiamina, riboflavina, niacina y $B$-caroteno y finalmente, la guayaba es una fruta de alto valor por su contenido de calcio y fósforo, vitaminas A, B1 (tiamina), B2 (riboflavina) y ácido ascórbico (Thuaytong y Anprung, 2011; Lopez et al., 2012; Zapata et al., 2013; Guimba et al., 2016). En Colombia, las recomendaciones de Ingesta de energía y nutrientes para individuos ha adoptado valores de referencia de ingesta adecuada (Al) para Fibra y de Aporte Dietético Recomendado (RDA) para Vitamina A, $\mathrm{C}$, hierro y calcio, tomando referencias de la FAO-OMS de 2001 para hierro y vitamina $A$ y del Consejo de Alimentación y Nutrición y el Instituto de medicina de los Estados Unidos (FNB:IOM) de 2010 y 2011 para vitamina $\mathrm{C}$ y calcio. El objetivo de este estudio fue evaluar técnicas de deshidratación para estabilizar y concentrar las fracciones comestibles de mango, guayaba y limón, como ingredientes nutracéuticas para incorporarse en otras matrices alimentarias. Esta investigación se realizó en el marco de un proyecto de contribución a la solución de las necesidades tecnológicas de pequeños productores agropecuarios del departamento del Atlántico.

\section{METODOLOGÍA}

El material vegetal mango (Mangifera indica), guayaba (Psidium guajava) y limón (Citrus limón), recién cosechado y seleccionado, procedente del municipio de Santa Lucía (Atlántico-Colombia), fueron remitidos al Instituto de Ciencia y Tecnología Alimentaria en Medellín, bajo cadena de frio. Los productos fueron recibidos, inspeccionados, lavados, desinfectados y posteriormente almacenados a $2-4^{\circ} \mathrm{C}$.

\section{Caracterización Composicional y fisicoquímica de las frutas}

Para la caracterización composicional de las frutas sin procesamiento se procedió a determinar el contenido de humedad $(\% \mathrm{H})$ mediante pérdida por desecación empleando una Termo balanza (Ohaus MB-200) a $105^{\circ} \mathrm{C}$. 
El contenido de Proteína (\%Pro) por digestión Kjeldahl, grasa total (\%Gra) por extracto etéreo, fibra cruda (\%FC) por método gravimétrico (digestión acido - base), cenizas (\%Cen) por método gravimétrico. Se determinaron de acuerdo con el método estándar oficial (AOAC, 2003). El contenido total carbohidratos (\%Cho) de las muestras se calculó restando el valor de proteína, grasa, fibra, ceniza y contenido de humedad de 100\%. En la determinación de los sólidos solubles se utilizó una lectura refractométrica (AOAC 932.12/90). La acidez se midió por el método de titulación potenciométrica y expresado como ácido cítrico (AOAC 942.05/90).

La determinación de Potasio $(\mathrm{K})$, Magnesio $(\mathrm{Mg})$, Calcio $(\mathrm{Ca})$ y Hierro $(\mathrm{Fe})$ se realizó de acuerdo al método AOAC 999.10. Esta metodología se basa en la destrucción de la materia orgánica mediante la adición de ácido nítrico concentrado, peróxido de hidrógeno y digestión ácida en recipiente cerrado asistida por horno microondas analítico. Posteriormente, se efectúa la cuantificación de cada analito por espectrofotómetro de absorción atómica con llama para el potasio, magnesio, calcio y hierro. La determinación de vitamina C (Vit C) se realizó mediante una extracción líquido-sólido con una mezcla de ácido metafosfórico al 3\% y ácido acético al 8\%, de esta solución se toma una alícuota y se afora con ácido acético al 0,1\%, la cuantificación se realizó con un cromatógrafo de líquidos acoplado a un detector de masas en tándem triple cuadrupolo. Los resultados se expresan en $\mathrm{mg} / 100 \mathrm{~g}$ de porción comestible. Todas las determinaciones anteriores se realizaron por triplicado.

\section{Deshidratación osmótica (DO)}

Se realizó en trozos de mango y guayaba, para lo cual se procedió a lavar y desinfectar la fruta con solución desinfectante a base de ácidos orgánicos y extractos de cítricos (Citrosan®) en concentración de 2,5 a 3,0 $\mathrm{ml} / \mathrm{L}$ equivalente a $900-1080 \mathrm{ppm}$, ambas frutas fueron peladas y se les retiraron las semillas, la pulpa fue cortada en trozos de $1 \times 1 \mathrm{~cm}$. La solución osmo deshidratadora se formuló con azúcar a una concentración $(40 \% \mathrm{p} / \mathrm{p})$, a la solución se le aplicó un proceso de calentamiento hasta $80^{\circ} \mathrm{C}$ y se enfrió rápidamente. Los cubos de la fruta se dispusieron en una bolsa para vacío (co-extruido de PA /LDPE en 70 micras de espesor transparente) y se agregó el jarabe anteriormente descrito en una relación de peso (2:1 jarabe / fruta). Se realizaron dos ciclos de vacío en una máquina de campana. El producto sellado se dejó en reposo a temperatura de refrigeración $\left(2-4^{\circ} \mathrm{C}\right)$ por 48 horas. Transcurrido este tiempo los trozos se escurrieron por gravedad usando un colador de acero inoxidable, fueron dispuestos en bandejas perforadas y se aplicó un secado a $40^{\circ} \mathrm{C}$ por 2 horas en horno de convección forzada. Se dejaron enfriar y se empacaron en bolsas (PET metalizado/PA/LDPE) y almacenadas en refrigeración $\left(2-4^{\circ} \mathrm{C}\right)$.

\section{Secado por aire caliente (SC)}

Se realizó en trozos de mango y guayaba, se adecuaron de la manera que la indicada para DO, los trozos fueron dispuesto en bandejas perforadas para someterse a secado a $50^{\circ} \mathrm{C}$ en horno de convección forzada por 12 horas o hasta alcanzar un nivel máximo de $10 \%$ de humedad, se dejaron enfriar y se procedió a reducir su tamaño en un molino de cizalla hasta una granulometría de malla Nro.100 U.S. Al limón se le realizó lavado y desinfección, se procedió a obtener la ralladura de la cascara del limón la cual fue secada a $50^{\circ} \mathrm{C}$, se extrajo el zumo de limón de manera manual, se mezcló con pulpa de mango en proporciones 90 mango $/ 10$ limón, se esparció en bandejas de PP y se sometió a deshidratación a $50^{\circ} \mathrm{C}$ por 12 horas, el producto obtenido es una lámina de fruta deshidratada.

\section{Caracterización nutricional de los productos deshidratados}

La caracterización se llevó acabo siguiendo las metodologías descritas por la AOAC (2003). La determinación de Calcio $(\mathrm{Ca})$ e Hierro $(\mathrm{Fe})$ se realizó de acuerdo al método AOAC 999.10. El contenido de fibra dietaría (\%FD) se determinó empleando el método enzimático AOAC 985.29. La actividad de agua (aw) se determinó a $25^{\circ} \mathrm{C}$ empleando el método AOAC 978.18. La determinación de humedad para ralladura de limón, lámina de mango-limón, mango en polvo y guayaba en polvo se realizó mediante Termo balanza (Ohaus MB-200) a $105^{\circ} \mathrm{C}$, mientras que el mango y la guayaba osmo-deshidratada fueron analizados según la NTC 572 , empleando un horno de convección forzada a $105^{\circ} \mathrm{C}$. La determinación de vitamina $C$ (Vit C) se realizó empleando el mismo método descrito para frutas. La determinación de vitamina $A$ (Vit $A$ ) se realizó por cromatografía líquida con detector de arreglo de diodos, en la preparación de la muestra se utilizó una saponificación con hidróxido de potasio en medio alcohólico, se adiciono ácido ascórbico como antioxidante antes de realizar un calentamiento por máximo 45 min a una temperatura entre 80 a $100^{\circ} \mathrm{C}$, después de este tiempo se dejó enfriar la muestra para realizar una extracción con n-hexano de 3 a 4 veces, se realizaron lavados con agua hasta obtener un $\mathrm{pH}$ neutro, la muestra se secó en roto evaporador, el residuo fue disuelto en fase móvil e inyectado en el HPLC. Los resultados se expresaron en UI/100g que corresponden a la actividad de $0,344 \mathrm{~g}$ de acetato de vitamina A puro cristalino. 
Para cálculos de aportes nutricionales se utilizaron los valores de IA para fibra de $25 \mathrm{~g} /$ día. RDA de Vitamina C de 60 mg/día, vitamina A de 2333 IU o 700 g/día, calcio 1000 mg/día y para hierro 18 mg/día.

\section{RESULTADOS Y DISCUSIÓN}

En la Tabla 1, se observan los valores obtenidos para el análisis composicional de guayaba, limón y mango, como se puede observar la mayor composición de las frutas es agua, seguido de carbohidratos, proteína, ceniza y grasa. El valor de grasa en la guayaba es mucho mayor comparado con el limón y mango, esto puede deberse principalmente al elevado contenido de semillas que puede estar entre $6-12 \%$ del peso total de la fruta, que a su vez está compuesta por un $16 \%$ de grasa (Anany, 2015). La mayor cantidad de carbohidratos se presentó en el mango el cual es una fruta caracterizada por su alto contenido en azúcares.

Tabla 1. Análisis composicional de frutas

\begin{tabular}{|l|c|c|c|}
\hline Producto & Limón & Mango & Guayaba \\
\hline \% Grasa & 0.24 & 0.07 & 8.48 \\
\hline \% Proteína & 1.36 & 1.34 & 0.68 \\
\hline \% Humedad & 84.71 & 73.94 & 84.10 \\
\hline \% Fibra Cruda & 0.75 & 0.86 & 0.52 \\
\hline$\%$ Cenizas & 0.65 & 0.49 & 0.72 \\
\hline$\%$ Carbohidratos & 13.04 & 24.16 & 5.02 \\
\hline $\mathrm{K}(\mathrm{mg} / 100 \mathrm{~g})$ & 147.57 & 134.42 & 224.21 \\
\hline $\mathrm{Mg}(\mathrm{mg} / 100 \mathrm{~g})$ & 8922 & 12.18 & 7966 \\
\hline $\begin{array}{l}\text { Ca } \\
\text { (mg/100g) }\end{array}$ & 60.69 & 15.05 & 15.85 \\
\hline Fe (mg/100g) & $<0.25$ & $<0.25$ & $<0.25$ \\
\hline $\mathrm{Vit} \mathrm{C}(\mathrm{mg} / 100 \mathrm{~g})$ & 13.48 & 44.09 & 41.59 \\
\hline
\end{tabular}

El valor hallado para Vitamina $C$ en guayaba fue de $41,59 \mathrm{mg} / 100 \mathrm{~g}$ de fruta fresca equivalentes al $69,3 \%$ del RDA para adultos. El contenido típico de Vitamina C oscila entre $50-400 \mathrm{mg} / 100 \mathrm{~g}$ de fruta fresca (Lopez et al., 2012; Ramirez y Pacheco de Delahaye, 2011; Restrepo-Sánchez, 2009). Un estudio realizado por Contreras et al. (2011) reportó un contenido de $257 \mathrm{mg} / 100 \mathrm{~g}$ en guayaba manzana (Hibrido de Psidium guajava) y $102 \mathrm{mg} / 100 \mathrm{~g}$ guayaba agria (Psidium araca) cultivadas en Colombia. El contenido de Vitamina C en mango fue de $44,09 \mathrm{mg} / 100 \mathrm{~g}$ de fruta fresca equivalentes al $73,4 \%$ del RDA. Estudios en distintas variedades de mango han reportado un rango de 41,75 a 124,40 mg de ácido ascórbico por 100 gramos de pulpa (Guimba et al., 2016; Zhang et al., 2018). El contenido de vitamina C hallado en el limón en este estudio fue de $13,48 \mathrm{mg} / 100 \mathrm{~g}$ de frutas fresca $(84,77 \mathrm{mg} / 100 \mathrm{~g} \mathrm{BS})$, mientras que en la literatura se han reportado valores entre 51,3-74,3 mg/100g de frutas fresca (Guimba et al., 2016). Los contenidos de Vitamina C y de muchos otros compuestos bioactivos pueden verse afectados por el genotipo, manejo pre y postcosecha, estado de madurez y operaciones de proceso y conservación (Mditshwa et al., 2017).

En cuanto al contenido de potasio, la fruta que mayor cantidad presentó fue la guayaba con 224,21 mg. El contenido de fibra cruda de las tres frutas no fue mayor al 1\%. En cuanto al contenido de minerales los niveles de calcio, potasio, hierro y magnesio son similares a los reportados en la Tabla de composición de alimentos del Centro de atención nutricional (Quintero y Escobar, 2001). La Tabla 2 muestra la caracterización fisicoquímica de las frutas empleadas en su estado de madurez de consumo, el valor de grados brix para el mango es similar al reportado (Alvis et al.,2016), el porcentaje de pulpa fue mayor en la guayaba, explicado por el menor peso de sus semillas.

Tabla 2. Parámetros fisicoquímicos de frutas

\begin{tabular}{|l|c|c|c|}
\hline Producto & $\begin{array}{c}\text { \%Acidez (gde ácido } \\
\text { cítrico/100g) }\end{array}$ & ${ }^{\circ}$ Brix & $\%$ Pulpa \\
\hline Limón & 6,40 & 7,2 & 35 \\
\hline Mango & 1,17 & 12,3 & 54 \\
\hline Guayaba & 0,46 & 7,4 & 63 \\
\hline
\end{tabular}

La Tabla 3, muestra los indicadores de los procesos de deshidratación aplicados a las frutas, donde $\Delta \% \mathrm{H}$ corresponde a los cambios en el contenido de humedad ocasionados por el proceso de deshidratación, $\Delta^{\circ}$ Brix a los cambios en el contenido de solidos solubles y $\Delta$ Peso a los cambios en el peso por cada $100 \mathrm{~g}$ de producto. 
En cuanto a las variaciones del contenido de humedad $(\Delta \% \mathrm{H})$, estas fueron mayores cuando se utilizó el proceso SC alcanzando valores superiores al $65 \%$, en contraste con el proceso de DO en la cual las variaciones de humedad no superaron el $23 \%$. Giraldo-Bedoya et al. (2004), tuvieron variaciones similares de sólidos solubles en fruta, al osmodeshidratar mora con jarabe de sacarosa de $70^{\circ}$ Brix y una relación 1:2 fruta: jarabe, sin pulsos de vacío. Las variaciones de peso entendidas como la merma de los procesos fueron menores.

Tabla 3. Indicadores de proceso de deshidratación

\begin{tabular}{|l|c|c|c|c|}
\hline Producto & Proceso & $\Delta \% H$ & $\Delta^{\circ}$ Brix & $\Delta$ Peso \\
\hline Lámina de Mango-Limón & SC & 71,6 & - & 90,1 \\
\hline Mango & SC & 67,5 & - & 91,3 \\
\hline Mango & DO & 22,4 & $-8,3$ & 9,6 \\
\hline Guayaba & SC & 78,2 & - & 92,2 \\
\hline Guayaba & DO & 21,4 & $-10,6$ & 5,4 \\
\hline
\end{tabular}

En la Tabla 4 se encuentran los resultados de contenido de humedad final y actividad de agua las frutas deshidratadas. Como se observa, los procesos de deshidratación, utilizados para la concentración de sustancias de interés nutraceútico, permiten obtener dos grupos de productos uno de humedad baja y otro de intermedia, estos últimos requirieron almacenamiento en refrigeración dada su actividad de agua. En cambio, los productos con actividad de agua baja, inferior a 0,5 son estables a temperatura ambiente siempre y cuando se envasen en materiales de alta barrera.

Tabla 4. Análisis fisicoquímico en productos deshidratados

\begin{tabular}{|l|c|c|}
\hline Producto & $\% H$ & $a_{w}$ \\
\hline $\begin{array}{l}\text { Ralladura de Limón } \\
\text { deshidratada }\end{array}$ & 7,99 & 0,492 \\
\hline Lámina de Mango-Limón & 3,25 & 0,321 \\
\hline Mango en polvo & 6,44 & 0,345 \\
\hline Guayaba en polvo & 5,85 & 0,287 \\
\hline Mango osmodeshidratado & 51,49 & 0,944 \\
\hline Guayaba osmodeshidratada & 62,68 & 0,962 \\
\hline
\end{tabular}

En la tabla 5 se encuentran recopilados los resultados de las sustancias de interés nutraceútico analizadas en el presente estudio. Se destacan los altos contenidos de fibra dietaría encontrada en Ralladura de Limón, Guayaba en polvo y Mango en polvo. Thuaytong y Anprung (2011) reportan una relación aproximada 1:3 de fibra soluble e insoluble en Guayaba, y estudiaron su potencial prebiótico frente a fibras comerciales como la Inulina. Por otra parte, el contenido de FD en mango en polvo no fue similar al reportado por otros autores, con un valor del $28 \%$ (Vergara-Valencia et al., 2007), dado que solo se empleó la pulpa para obtener el polvo con el propósito de evitar defectos sensoriales en el color y sabor del producto. En cuanto a la Ralladura de cáscara de Limón, se obtuvo un alto valor en el contenido de Fibra y Calcio, alcanzando en este último un valor de 2066,01 mg/100g, equivalente al $200 \%$ del RDA de calcio.

Tabla 5. Análisis compuestos de interés nutracéutico en productos deshidratados

\begin{tabular}{|l|c|c|c|c|c|}
\hline \multicolumn{1}{|c|}{ Producto } & $\% F D$ & $\begin{array}{c}\mathrm{Ca} \\
(\mathrm{mg} / 100 \mathrm{~g})\end{array}$ & $\begin{array}{c}F e \\
(\mathrm{mg} / 100 \mathrm{~g})\end{array}$ & $\begin{array}{c}\text { Vit C } \\
(\mathrm{mg} / 100 \mathrm{~g})\end{array}$ & $\begin{array}{c}\text { Vit A } \\
(\text { UI })\end{array}$ \\
\hline Ralladura de Limón & 45.27 & 2066.01 & 1.93 & $<1.87$ & $<2.0$ \\
\hline Lámina de Mango-Limón & 6.32 & 54.26 & $<0.25$ & $<1.87$ & 2.3 \\
\hline Mango en polvo & 10.98 & 46.17 & 4.42 & 87.21 & 2.1 \\
\hline Guayaba en polvo & 44.44 & 65.56 & 0.93 & 32.14 & 3.6 \\
\hline Mango osmodeshidratado & 2.39 & 15.97 & 2.04 & $<1.87$ & 2.8 \\
\hline $\begin{array}{l}\text { Guayaba } \\
\text { osmodeshidratada }\end{array}$ & 7.25 & 15.82 & $<0.25$ & $<1.87$ & $<2.0$ \\
\hline
\end{tabular}

El contenido de Vitamina $C$ en el mango y guayaba en polvo deshidratados por aire caliente alcanzaron valores de 87,21 y $32,14 \mathrm{mg} / 100 \mathrm{~g}$, equivalentes a 93,21 y $34,14 \mathrm{mg} / 100 \mathrm{~g}$ BS. Si se toman los valores del contenido de Vitamina $\mathrm{C}$, expresados en base seca, comparativamente con los obtenidos en el material fresco 
se calcula una pérdida del nutriente del $88 \%$ en guayaba y de $42 \%$ para el mango, una sensibilidad similar fue reportada por Serpa-Guerra et al. (2015) en Guayaba-pera a la misma temperatura de secado, la cual alcanzo un $80 \%$. De manera similar se reportó para Ají secado bajo convección con aire caliente a $50^{\circ} \mathrm{C}$, una perdida aproximada del $83 \%$ de Vit C (Vega-Galvez et al., 2009). A pesar de lo anteriormente expuesto, el proceso de deshidratación actúa como un proceso de concentración de la cantidad remanente de vitamina C.

Sin embargo, el contenido de Vitamina $C$ en los productos deshidratados osmóticamente disminuyó drásticamente. Estas pérdidas pueden ocasionarse debido a que durante la osmo deshidratación, se establece una transferencia de masa de dos vías: el agua y algunas sustancias naturales solubles (azúcares, vitaminas, pigmentos, ácidos orgánicos, sales minerales) fluyen de la fruta hacia la solución osmótica, y en la dirección opuesta, los solutos solubles se pueden transferir desde la solución al fruto (Chandra y Kumari, 2015; Tortoe, 2010). Pérdidas significativas de vitamina $C$, han sido reportadas durante el proceso de osmo deshidratación de Uchuva al pasar de 92 a $34 \mathrm{mg} / 100 \mathrm{~g}$ de fruta (Molina y Orjuela, 2013). La vitamina A en los materiales vegetales deshidratados, presentó valores muy bajos, Chen et al. (2007) han reportado el efecto de las técnicas de secado, especialmente el uso de aire caliente sobre la degradación de carotenoides en mango. Esto además se pudo deber a que el mayor contenido de carotenoides en mango se encuentra en la cáscara (Ajila et al., 2010; Ribeiro et al., 2007) la cual no fue usada en este estudio.

\section{CONCLUSIONES}

La técnica de deshidratación osmótica resulta una alternativa adoptable por los pequeños productores para mantener un suministro constante de ingredientes nutracéuticos en productos de pastelería o panadería. Si bien, durante la deshidratación osmótica se produce la degradación de algunos nutrientes del alimento, presenta la ventaja de poder agregar minerales y vitaminas a la solución de deshidratación para que luego ingresen al producto final enriqueciéndolo en nutrientes.

\section{REFERENCIAS}

Ahmed, I., I. M. Qazi y S. Jamal, Developments in osmotic dehydration technique for the preservation of fruits and vegetables, doi:10.1016/j.ifset.2016.01.003, Innovative Food Science and Emerging Technologies (en linea), 34, 29-43, (2016)

Ajila, C. M., L. J. Rao y U. J. S. P. Rao, Characterization of bioactive compounds from raw and ripe Mangifera indica $L$. peel extracts, doi: 10.1016/j.fct.2010.09.012, Food and Chemical Toxicology (en linea), 48 (12), 3406-3411, (2010)

Alvis-Bermudez, Armando, García-Mogollon, Carlos, \& Dussán-Sarria, Saúl. Cambios en la Textura y Color en Mango (Tommy Atkins) PreSecado por Deshidratación Osmótica y Microondas. (En línea: https://dx.doi.org/10.4067/S071807642016000200005. Acceso: 18 de enero 2018). Información tecnológica, 27(2), 31-38, (2016).

Anany, E. A. M. Nutritional composition, antinutritional factors, bioactive compounds and antioxidant activity of guava seeds (Psidium Myrtaceae) as affected by roasting processes, doi: 10.1007/s13197-013-1242-1, Journal of Food Science and Technology (en linea), 52(4), 2175-2183, (2015)

AOAC. Official methods of analysis (17th ed). Gaithersburg, MD, EU, (2003)

Chandra, S. y D. Kumari, Recent Development in Osmotic Dehydration of Fruit and Vegetables: $A$ Review, doi: 10.1080/10408398.2012.664830, Critical Reviews in Food Cience and Nutrition (en linea), 55 (4), 552-561, (2015)

Chen, J., C. Tai y B. Chen, Effects of different drying treatments on the stability of carotenoids in Taiwanese mango (Mangifera indica L.), doi: 10.1016/j.foodchem.2005.10.056, Food Chemistry (en linea), 100 (3), 1005-1010, (2007)

Contreras-Calderón, J., L. Calderón-Jaimes. y otros autores, Antioxidant capacity, phenolic content and vitamin C in pulp, peel and seed from 24 exotic fruits from Colombia, doi: 10.1016/j.foodres.2010.11.003, Food Research International (en linea), 44 (7), 2047-2053, (2011)

Diaz, A., H. Bolivar. y otros autores, Productos de Confitería nutracéutica: una opción empresarial para cultivadores de frutas $y$ hortalizas, $1^{\mathrm{a}}$ edicion, ISBN 978-958-5430-23-5, (en linea: http://publicaciones.unisimonbolivar.edu.co/edicionesUSB/, Acceso: 09 de Septiembre 2017), Ediciones Universidad Simón Bolivar, Barranquilla -Atlantico, Colombia, (2017)

Erenturk, S., M. S. Gulaboglu, y S. Gultekin, The effects of cutting and drying medium on the vitamin C content of rosehip during drying, doi: 10.1016/j.jfoodeng.2004.07.012, Journal of Food Engineering (en linea) 68, 513-518, (2005)

Fernández, D., S. Muñiz. y otros autores. Cinética de secado de fruta bomba (Carica papaya L., cv. Maradol Roja) mediante los métodos de deshidratación osmótica y por flujo de aire caliente, (En línea http://scielo.sld.cu/scielo.php?script=sci_abstract\&pid=S2071-00542015000100003, acceso: 12 de septiembre 2017), ISSN 2071-0054, 24(1), Revista Ciencias Técnicas Agropecuarias, 22-28, (2015)

Giraldo B. D. P., V. L. M. Arango y C. C. J. Márquez., Osmodeshidratación de mora de castilla (Rubus glaucus Benth) con tres agentes edulcorantes. (en linea http://www.scielo.org.co/pdf/rfnam/v57n1/a08v57n1.pdf?, acceso: 13 de septiembre 2017), Revista Facultad Nacional de Agronomía-Medellín, 57, 1-18, (2004) 
Guimba, I., L. Ahrné. y otros autores, Retention of $\beta$-carotene and vitamin $C$ in dried mango osmotically pretreated with osmotic solutions containing calcium or ascorbic acid, doi: 10.1016/j.fbp.2016.02.010, Food and Bioproducts Processing, 98(1), 320-326, (2016).

Lopez, R., M. Dupas. y otros autores, Tropical and Subtropical Fruits Postharvest Physiology, Processing and Packaging, $1^{a}$ edición, 469-477. Jhon Wiley \& Sons, Inc., New Delhi, India (2012)

Mendoza-Corvis, Fernando A, Hernández, Elvis J, \& Ruiz, Luis E. Efecto del Escaldado sobre el Color y Cinética de Degradación Térmica de la Vitamina $C$ de la Pulpa de Mango de Hilacha (Mangífera indica var magdalena river). (En línea: https://dx.doi.org/10.4067/S0718-07642015000300003. Acceso: 18 de enero 2018). Información tecnológica, 26(3), 0916, (2015)

Mditshwa, A., L. S Magwaza y otros autores, Postharvest factors affecting vitamin $C$ content of citrus fruits: $A$ review, doi: 10.1016/j.scienta.2017.02.024, Scientia Horticulturae, 218(1), 95-104, (2017).

Molina, C. B. y O. C. Orjuela., Cambios en el contenido nutricional de la uchuva (Physalis peruviana) frente a osmodeshidratación como método de conservación. (en linea https://aprendeenlinea.udea.edu.co/revistas/index.php/nutricion/article/view/18996, acceso: 13 de septiembre 2017), ISSN 2248-454X, Perspectivas En Nutrición Humana, 15(2), 149-156, (2013)

Pereira, A. G., S. M. Becerá. y otros autores, Análisis comparativo de la cinética de deshidratación Osmótica y por Flujo de Aire Caliente de la Piña (Ananas Comosus, variedad Cayena lisa). (en linea: http://scielo.sld.cu/scielo.php?pid=S2071 00542013000100011\&script=sci_arttext\&tIng=en, acceso: 13 de septiembre 2017), ISSN 2071-0054, Revista Ciencias Técnicas Agropecuarias, 22(1), 62-69, (2013)

Pérez L, C. (2006). Nutracéuticos: componente emergente para el beneficio de la salud. (en linea: http://www.redalyc.org/pdf/2231/223120665003.pdf Acceso: 14 de septiembre 2017), ISSN 0138-6204, ICIDCA. sobre los derivados de la caña de azúcar, 3, 20-28, (2006)

Quintero, S. D. y L. Escobar, Tabla de composición de alimentos. Centro de Atención Nutricional. $2^{\mathrm{a}}$ edición, Medellín, Colombia (2001)

Ramirez, A. y E. Pacheco de Delahaye, Composición química y compuestos bioactivos presentes en pulpas de piña, guayaba y guanábana. (en linea: http://www.redalyc.org/pdf/339/33917727011.pdf. Acceso: 12 septiembre (2017), ISSN 0378-1844, Interciencia, 36(1), 71-75, (2011)

Restrepo-Sanchez, D. C., C. E. Narvaez-Cuenca y L. P. Restrepo-Sanchez, Extracción de compuestos con actividad antioxidante de frutos de guayaba cultivada en Velez-santander, Colombia. doi: 10.1590/S0100-40422009000600030, Quimica Nova (en linea), 32(6), 1517-1522, (2009)

Ribeiro, S., J. Queiroz y otros autores, Antioxidant in Mango (Mangifera indica L.) Pulp, doi: 10.1007/s11130-006-0035-3, Plant Foods for Human Nutrition (en linea), 62, 13-17, (2007)

Serpa-Guerra, A. M., D. C. Vásquez-Osorio, y otros autores, Comparison of two dehydration techniques for "pear" guava (Psidium guajava I.) on the effects of the vitamin $C$ content and on the behavior of the technical and functional properties of the dietary fiber, (en linea: http://www.scielo.org.co/scielo.php?pid=S179444492015000100002\&script=sci_arttext\&tIng=en. Acceso: 14 de septiembre 2017), ISSN: 1794-4449, Revista Lasallista de Investigación, 12(1), 10-20, (2015)

Thuaytong, W. y P. Anprung, Bioactive compounds and prebiotic activity in Thailand-grown red and white guava fruit (Psidium guajava L.), doi: 10.1177/1082013210382066, Revista de Agroquímica y Tecnología de Alimentos (en línea), 17(3), 205-212, (2011)

Tortoe, C., A review of osmodehydration for food industry. (en linea: http://www.academicjournals.org/ajfs. Acceso: 12 de septiembre 2017), ISSN 1996-0794, African Journal of Food Science, 6, 303-324 (2010)

Vega-Galvez, A., R. Lemus-Mondaca. y otros autores, Effect of air-drying temperature on physico-chemical properties, antioxidant capacity, colour and total phenolic content of red pepper (Capsicum annuum, L. var. Hungarian), doi: 10.1016/j.foodchem.2009.04.066, Food Chemistry (en linea), 117, 647-653, (2009)

Vergara-Valencia, N., E. Granados-Pérez y otros autores. Fibre concentrate from mango fruit: Characterization, associated antioxidant capacity and application as a bakery product ingredient, doi: 10.1016/j.Iwt.2006.02.028, LWT-Food Science and Technology (en linea), 40(4), 722-729, (2007)

Zapata, K., F. B. Cortes y B. A. Rojano, Polifenoles y Actividad Antioxidante del Fruto de Guayaba Agria (Psidium araca), doi: 10.4067/S0718-07642013000500012, Información Tecnológica (en línea), 24(5), 103-112, (2013)

Zhang, Y., J. H. Zhao y otros autores, Changes in the vitamin $\mathrm{C}$ content of mango with water state and ice crystals under state/phase transitions during frozen storage, doi: 10.1016/j.jfoodeng.2017.11.003, Journal of Food Engineering, 222(1), 49-53, (2018). 
